\title{
Intergenerational Pathways from Reflective Functioning to \\ Infant Attachment Through Parenting
}

\author{
Karin Ensink ${ }^{1}$ \\ Lina Normandin ${ }^{1}$ \\ André Plamondon ${ }^{2}$ \\ Nicolas Berthelot ${ }^{3}$ \\ Peter Fonagy ${ }^{4}$
}

Authors Note

1. École de psychologie, Université Laval, Québec, Canada

2. Department of Educational Science, Université Laval, Québec, Canada

3. Université du Québec Trois-Rivières, Québec, Canada

4. University College London, London, England

This research was supported in part by grants to Karin Ensink and Lina Normandin from the Fonds québécois de la recherche sur la société et la culture, from the Social Sciences and Humanities Research Council of Canada and from the Centre de recherche interdisciplinaire sur les problèmes conjugaux et les agressions sexuelles. Correspondence concerning this article should be addressed to Karin Ensink, École de psychologie, 2325, rue des Bibliothèques, Québec, QC Canada )G1V 0A6; E-mail: Karin.Ensink@psy.ulaval.ca 


\begin{abstract}
The aim of this prospective study was to examine temporal pathways from mothers' reflective functioning through parenting to infant attachment measured more than 16 months later. Participants were 88 motherinfant dyads from demographically diverse backgrounds and including a group of mothers with histories of childhood maltreatment. Reflective Functioning was assessed using the RF rating of the Adult Attachment Interview before the birth of the baby. Parenting was assessed when the infants were 6 months old using the Maternal Sensitivity Scale, as well as when they were 16 months using the Disconnected and Extremely Insensitive Parenting Scale. Infant attachment was assessed when the infants were 16 months old using the Strange Situation. As hypothesized, the study findings showed that mothers' mentalization regarding their own early attachment relationships was associated with later parenting and infant attachment. Negative parenting behaviours explained the link between mothers' RF about their own attachment relationships and both infant attachment disorganization and infant attachment insecurity. The findings suggest that mothers' mentalization about their early attachment relationships has important implications in the transition to becoming parents themselves. Mentalization appears to be particularly important in helping mothers screen and inhibit negative parenting behaviours that would otherwise undermine infant attachment security and organization.
\end{abstract}

Keywords: reflective functioning, mentalization, maternal sensitivity, parenting, infant attachment 


\section{Intergenerational Pathways from Reflective Functioning to Infant Attachment Through Parenting}

In a now classic study, Fonagy, Steele, Steele, Moran and Higgitt (1991) showed that mothers' capacity to mentalize about their own early attachment experiences measured using the Adult Attachment Interview predicted infant attachment security more than 16 month later. Based on these findings, Fonagy and colleagues (1991) proposed that mentalization, the uniquely human capacity to be cognizant of one's own affects and imagine the feelings and thoughts of others, plays an important role in the intergenerational transmission of attachment. An intergenerational pattern is proposed where the parent's mentalization and capacity to imagine what the infant's behavior says about what they feel, need and want, underlies sensitive responding and promotes attachment security (Fonagy \& Target, 1997). The development of mentalization in turn is seen as rooted in attachment, with infants discovering their minds through being in a relationship with someone who treats them as someone with a mind. However, the findings of Fonagy and colleagues (Fonagy, et al., 1991) of an intergenerational pattern where mothers' mentalization about their own early attachment relationships is linked to infant attachment, have not been replicated. Also surprisingly, no study has examined the link between mothers' mentalization regarding their own attachment relationships and parenting, and most research has focused on parental RF about the child using the Parent Development Interview (PDI: Slade et al., 2005). Mothers' mentalization about their own parents and attachment relationships is likely to be particularly important in the transition to becoming parents themselves, and it would seem a matter of priority to examine this empirically. The aim of this study was to examine evidence for the theoretically assumed intergenerational pathway from mothers' RF regarding attachment though parenting to infant attachment. 


\section{Reflective functioning, parenting and infant attachment}

Reflective functioning is seen as underlying sensitive responding by helping mothers to mentally put themselves in the place of the infant and imagine the infant's experience (Fonagy \& Target, 1997). By the same token, RF can be expected to play a role in inhibiting negative parenting by helping parents mentally step back and be cognizant of their own affects and their impact on the infant. To date, only two studies have examined the relationship between parental RF about the child using the PDI, parenting, as well as infant attachment. Grienenberger et al. (2005) demonstrated in a high income sample $(n=48)$ that higher parental RF was related to less disrupted affective communication in interaction with their infants as assessed with the Atypical Maternal Behaviour Instrument for Assessment and Classification (AMBIANCE) (Lyons-Ruth, Bronfman, \& Parsons, 1999). These parenting behaviours mediated the relationship between parental RF and infant attachment, considered ordinally as secure, insecure and disorganized. More recently, Stacks and colleagues (2014), in a demographically diverse sample $(n=83)$ including mothers with histories of abuse and neglect, found that parental RF about the child, and not histories of abuse, was associated with parenting sensitivity, as well as negative parenting, and infant attachment security. However, neither parental RF, nor parenting, was related to infant attachment disorganization. Furthermore, the relationship between parental RF and infant attachment security was mediated by parenting sensitivity, but a similar path was not found for disorganization. The findings of other studies in which parental RF and parenting were examined are not all convergent. Schechter and colleagues (2008) found no relationship between parental RF and atypical behaviour in a referred sample of mothers exposed to violence, and neither did Pajulo and colleagues (2012) in a sample of drug dependent mothers in treatment. Suchman, Decoste, Leigh, and Borelli (2010) also did not find a relationship between parental 
RF in and maternal sensitivity, but mentalization regarding self was related to both sensitivity and socio-emotional and cognitive growth fostering. In addition, mothers' RF about past trauma was found to predict infant attachment disorganization (Berthelot et al., 2015) in a sample where all mother had histories of abuse and neglect. Although definite conclusions cannot be made based on these studies given that the majority had small sample sizes, it does suggest that the links between other dimensions of RF, parenting and attachment should be considered.

\section{Sensitive and Negative Maternal Behaviours and Infant Attachment}

In their seminal work on attachment assessment, Ainsworth and colleagues (Ainsworth, Blehar, Waters, \& Wall, 1978) proposed that the mother's awareness of the infant's communication and capacity to empathically identify and put herself "in the infant's shoes" is central to understanding the infant and responding in a well-timed, appropriate manner. Consistent with this, there is converging evidence that mothers of secure infants are more responsive to infant's signals (Ainsworth, et al., 1978; Isabella, 1993). By comparison, the interactional antecedents of disorganization, characterized as a "contradiction or an inhibition of action as it is being undertaken" (Main \& Hesse, 1990, p. 173), remain less clear (Beebe \& Steele, 2013). Initially high rates of disorganized attachment were observed in contexts of child abuse and was linked to threatening and fear inducing behaviours, thought to place the infant in an impossible situation of fear without resolution given that the only potential source of comfort is also the source of fear (Hesse \& Main, 2000; Main \& Hesse, 1990). Subsequently it was found that approximately $16 \%$ of infants in low risk samples also manifest disorganized attachment and this was associated with unresolved maternal states of mind regarding loss or trauma and threatening, frightened, dissociative, deferential sexualized, and disorganized/disorientated maternal behaviours ((Main \& Hesse, 1990; van IJzendoorn, 1995; 
van IJzendoorn \& Bakermans-Kranenburg, 2006). Expanding on this system, Lyons-Ruth and colleagues (Lyons-Ruth, et al., 1999; Lyons-Ruth \& Spielman, 2004) identified a range of atypical maternal behaviours that contribute to disorganization, including affective communication errors, intrusiveness or negative behaviours and withdrawal (measured with the AMBIANCE). Disrupted affective communication, for example when the mother laughs when an infant cries or fails to respond when he falls, predicted attachment disorganization in three studies (Beebe et al., 2010; Lyons-Ruth, et al., 1999; Madigan, Moran, \& Pederson, 2006), suggesting that it may be as important as the intrusion of the mother's aggression, fear and anxiety.

Reviewing the origins of disorganization, Bernier and Meins (2008) conclude that both disconnected (i.e. threatening and atypical) and insensitive behaviours contribute to disorganization, and that insensitive behaviours alone may predict disorganization especially in contexts with multiple risk factors, depending on the individual susceptibility of children. Recently, Out, Bakermans-Kranenberg and van IJzendoorn (2009) developed the DIP with the explicit purpose of facilitating the assessment of both disconnected behaviours and extremely insensitive behaviours, considered to represent complementary pathways to infant attachment disorganization.

\section{Histories of Abuse and Parenting}

Parental histories of abuse and neglect is a risk factor associated with compromised parenting capacity (Koren-Karie, Oppenheim, \& Getzler-Yosef, 2008). However, there are few studies of mother-infant interactions in samples where mothers have experienced childhood abuse, and some findings are somewhat unexpected. For example, Stacks and colleagues (2014) recently failed to find a relationship between abuse and the quality of parenting in interaction 
with infants. Abuse was not related to RF and while abuse was associated with PTSD and depression, it was not related to the quality of mother-infant interactions. This suggests that many pieces of the puzzle regarding the relationship between histories of abuse and parenting remain unsolved.

The first aim of the current study was to examine relationships between RF, parenting, and infant attachment. Because we were interested in associations with personal risk factors such as childhood histories of abuse, and demographic risk factors such as low education a relatively heterogeneous sample was recruited in terms of the presence of histories of abuse, education and income. To address this aim, RF was assessed prenatally and then parenting and attachment at 6 and 16 months post-partum. We hypothesized that higher RF would be related to more sensitive parenting and less parenting negativity and to secure and organized infant attachment. Furthermore, we expected that abuse and higher demographic risk would be associated with lower RF, lower sensitivity, higher parental negative behaviours and a higher prevalence of infant attachment disorganization.

The second aim was to examine pathways from mothers's mentalization via parental behaviours to infant attachment security and organization controlling for personal and demographic risk factors. We predicted that while RF associated maternal sensitivity would promote attachment security in the infant, low RF would be associated with more disconnected and intrusive aggressive behaviours, which in turn would be associated with an increased risk of attachment disorganization. 


\section{Method}

\section{Participants and Procedure}

Mothers were aged between 21 and $43(M=30.82, S D=4.00)$ and 64\% were primiparous mothers. All the participants were Caucasian, largely reflecting the ethnic composition of the French-Canadian city where the study was conducted. In terms of marital status, $66 \%$ were in common-law relationships, $32 \%$ were married and $2 \%$ were single. Regarding education, $14 \%$ had high-school diplomas, 30\% had college degrees and $56 \%$ had university degrees. In terms of annual income, $21 \%$ of families had household incomes ranging from $30000 \$$ to $60000 \$, 47 \%$ had incomes ranging from $60000 \$$ to $99999 \$$, and $32 \%$ had incomes over $100000 \$$ annually. Infant gender was equally presented and $50 \%$ of the infants in the sample were male.

In terms of histories of abuse, $30 \%$ of mothers reported histories of physical, sexual or emotional abuse; 11 reported physical abuse, 2 reported sexual abuse, 7 psychological abuse, 6 reported both sexual and physical abuse, and one reported sexual, physical, and psychological abuse.

Data reported here were collected as part of a larger longitudinal study designed to examine the transition from pregnancy to maternity. A total of 115 mothers participated in the current study at the prenatal assessment, 99 mother-infant dyads participated in the laboratory visit when their infants were 6 months old and 88 when the infants were 16 months old. Analysis in the current study is based on the 88 mother-infant dyads who participated in the 16 month assessment.

Pregnant women were recruited through flyers at community health services, at gynecological and obstetric clinics, as well as at commercial outlets for mothers and babies. 
Women interested in participating in the study were screened via telephone for histories of childhood maltreatment in order to recruit a ratio of approximately $30 \%$ of women exposed to childhood abuse so that the sample would be broadly representative of the reported prevalence of childhood maltreatment in the general population (Afifi et al., 2011; Scher, Forde, McQuaid, \& Stein, 2004). Exclusion criteria included age below 19, abnormal pregnancy, major psychosis, drug or alcohol abuse, and illiteracy or intellectual limitations. Mothers with complications during the last phases of the pregnancy, and with infants born very prematurely or with severe health problems were excluded from the study; sixteen dyads were therefore excluded from the initial sample.

\section{Measures and Procedure}

During the third trimester of their pregnancy women completed the Attachment Interview (AAI; George, Kaplan, \& Main, 1985) to assess their reflective functioning regarding their early attachment experiences. They subsequently returned to the laboratory with their infants twice. When the infants were 6 months old, maternal sensitivity assessed with the Sensitivity Scale (Ainsworth, Bell, \& Stayton, 1974) during filmed mother infant interactions where mothers played with their infants with and without toys, fed their infants, changed their nappies, filled out a questionnaire and engaged in an interview regarding the infant. When the infants were 16 months old, mothers and infants participated in the Strange Situation (SS; Ainsworth, et al., 1978) to assess mother-infant attachment as well as negative parenting using the Disconnected and Extremely Insensitive Behaviours Scale (DIB; Out, BakermansKranenberg and van IJzendoorn (2009). 


\section{Measures}

RF Coding of Adult Attachment Interview. Reflective functioning was rated from transcribed AAI's (George, et al., 1985). The AAI is a semi-structured interview designed to elicit the participant's current state of mind regarding attachment experiences with parents and other significant caregivers during childhood. The interview includes questions about the quality of childhood experiences with parents; the participant's responses to experiences of rejection, separation, loss, and trauma during childhood; and the participant's evaluation of the effects of those childhood experiences on his or her current functioning.

The AAI was coded for RF using the RF manual (Fonagy et al., 1998). The psychometric properties detailed in the manual include high inter-rater reliability as well as good discriminant validity and predictive validity across a number of samples. RF is rated on a scale of -1 to 9 with every score representing a different level of mental state explanation. Ratings of -1 indicate an attack on mentalization. Ratings of 0 indicate a refusal to engage in mentalization. Ratings of 1 indicate the absence of any recognition of mental states so that interpersonal reactions are described only in behavioural terms or individuals are described only in terms of physical or global characteristics. Ratings of 3 indicate limited ability to identify and acknowledge simple mental states and affects at a very general level, but without understanding how mental states function. Ratings of 5 indicate a basic capacity to consider how mental states influence behaviour and perceptions and are in turn evoked in interpersonal interactions. Higher ratings indicate increasingly full and sophisticated mental state accounts of subjective experience underlying interactions and ratings of 9 are reserved for exceptional accounts that reflect nuanced understanding of motivations underlying behaviour or unusual insights. RF is typically rated based on all AAI questions that explicitly demand and appreciation of mental states (e.g. 
'why did your parents behave as they did during your childhood?'). An overall RF score that represents respondents' characteristic level of RF is derived based on the individual scores using a decision algorithm outlined in the manual and that takes into account the frequency of low scores or failures in mentalization as well as the frequency of high scores

Two graduate students who were trained by the study authors rated the AAI protocols for reflective functioning. Inter-rater reliability was assessed on 19 coding protocols and the intraclass correlation coefficient was high $(I C C=.80)$ indicative of satisfactory inter-rater reliability.

Assessment of Reported Histories of Abuse. Potential participants were asked screening questions regarding the presence of childhood experiences of abuse during a telephone interview to assure that the sample would be composed of approximately $30 \%$ of mothers with such histories. To further assess the presence of reported histories of abuse, the information provided by the mothers during the AAI in response to questions regarding past traumatic experiences was used. Abuse history was defined as the mother's report of abuse on the AAI. This procedure has been used in previous studies and has been shown to be reliable in studies using the AAI as well as other independent measures of childhood abuse and neglect (Ensink, Berthelot, Bernazzani, Normandin, \& Fonagy, 2014).

Strange Situation Procedure. The Strange Situation (Ainsworth, et al., 1978) was used to assess infant attachment security and organization when infants were approximately 16 months old. The SS is widely used and its validity has been demonstrated in major long term studies (Sroufe, Egeland, Carlson, \& Collins, 2005). It consists of eight three minute episodes during which the mother leaves the infant (separation episodes) and reunites with the infant (reunion episodes). Two trained coders rated Strange Situations for the three organized 
attachment classifications (secure, anxious-resistant-insecure and anxious-avoidant-insecure) using the criteria of Ainsworth (Ainsworth, et al., 1978) and attachment disorganization using the criteria of Main and Solomon (Main \& Solomon, 1990). In this sample, 50\% were double coded with near full agreement for the organized attachment classifications and 97\% agreement for infant attachment disorganization.

Maternal Sensitivity. The Sensitivity Scale of Ainsworth (Ainsworth, et al., 1974) was used to code maternal sensitivity during mother infant interactions in the laboratory when the infants were 6 months old. Mothers were filmed while playing with their infants with and without toys, breast - or bottle fed their infants, changed their nappies, filled out a questionnaire and engaged in an interview regarding the infant. The first five minutes of each activity was coded, 30 minutes in total. The tasks were selected to be broadly representative of a range of everyday contexts in which mothers interact with their infants, with the last two contexts thought to resemble more stressful everyday contexts where the mother is required to divide her attention and keep the infant and mind while addressing the competing demands of another task or interacting with someone else. Sensitivity was rated on a continuous scale from 1 to 9 , with a 9 representing a very sensitive mother. Four dimensions of sensitivity are assessed, namely (a) awareness of the infant's communication, (b) an accurate interpretation of the signals, (c) appropriateness of reactions and (d) rapidity of the response to the infant's signals.

Sensitivity was rated by three trained graduate students who demonstrated high inter-rater reliability (ICC's from .90 to .98 ) on $25 \%$ of the sample. To deal with the negative skew in this variable, scores were reflected and log transformed.

Disconnected and Extremely Insensitive Parenting. The DIP was developed to assess both disconnected behaviour and extremely insensitive behaviour. For disconnected behaviour, 
all items from Main et al.'s (Main \& Hesse, 1998) coding instrument for frightening, frightened, dissociated, sexualized and disorganized parental behaviour were adapted to assess the following five dimensions of parental behaviour: 1) frightening and threatening behaviour, 2) behaviours indicating fear of the child, 3) dissociative behaviours indicative of absorption (freezing or handling as though the child is an inanimate object) or intrusion of an altered state of awareness (such as inexplicable shifts in mood or sudden fear regarding the environment), 4) interacting with the child in a timid, submissive and/or deferential manner, sexualized/romantic behaviours, 5) disoriented/disorganized behaviours (such as contradictions in behaviour or vocalizations, disorientation and other anomalous movements and postures). For disconnected behaviours, their sudden occurrence, lack of meta signals indicating play or affection (such as smiling) and lack of explanation for their occurrence are important considerations and scores are assigned for specific behaviours using clearly specified criteria (Out, et al., 2009).

For the second dimension, that of extreme parental insensitivity, two types are rated separately: 1) parental withdrawal and neglect; and 2) intrusive, negative, aggressive or otherwise harsh parental behaviours (Out, et al., 2009). This dimension is an adaptation of items from the AMBIANCE measure developed by Lyons-Ruth and colleagues (1999). The AMBIANCE items referring to extremely insensitive behaviour were combined into more general categories of parental behaviours. In the DIP, parental withdrawal/neglect is scored when the parent (repeatedly) fails to show responsive behaviour when the child is in distress, seeks contact or approaches the parent (Out et al., 2009). For example, the parent does not respond to the child's repeated vocalizations or ignores a crying child, or does not intervene when the child engages in dangerous behaviour. Physical intrusiveness is scored when the parent gets too close to the child and intrusively overrides the child's cues, (e.g. ignores the child's evident interest in 
a particular toy and attempt to physically move closer to the toy and engage with it, by either removing the toy, introducing another toy or picking the child up and physically changing their direction or position so they cannot engage with the toy) or engages in too intense and vigorous interactions. The distinction between extreme insensitivity and ordinary insensitive responses lies in the duration, frequency, quality and severity of the behaviours (e.g., aggressive behaviours) as well as the context in which the behaviour occurs (e.g., when the child is in distress).

The DIP has been shown to have good psychometric properties (Out, et al., 2009). Discrete disconnected and extremely insensitive behaviours were rated on a 9-point scale every time they occurred. For all dimensions, a final score was assigned, equal to the highest individual score or one point higher when the parental behaviour was severe or occurred frequently. In the present study the DIP was rated by two graduate students who were trained in consultation with the developers of the measure, and the intraclass correlation coefficient for the global scores was high, both for disconnected $(r=.92)$ and insensitive $(r=.79)$ behaviours.

We use the term negative maternal behaviours when referring to insensitive behaviours as assessed with the DIP so as not to contribute to confusion regarding the differences between sensitivity and insensitivity

\section{Analytic plan}

To address the first research objective, correlations between variables were examined and regressions and t-tests were used. To address the second research objective, a path analysis was conducted in Mplus 7.0 (L. K. Muthén \& Muthén, 1998-2012). Using this method, it is possible to test the hypothesis that reflective functioning is associated with outcomes through maternal behaviours while controlling for factors that might account for these associations. The models 
were estimated using a statistical method allowing for binary outcomes, means and variance adjusted Weighted Least Square (WLSMV) (B. O. Muthén, 2010). Regressions predicting the binary outcomes (i.e., secure/insecure (including disorganised) as well as organization/disorganization) were conducted using the probit link. This estimator was chosen because it can be used to derive indirect effects when using binary outcomes (L. K. Muthén \& Muthén, 1998-2012). The hypothesized indirect association between reflective functioning and child outcomes (attachment organization and attachment security) through maternal behaviour was tested by investigating the significance of the indirect pathways via both sensitive behaviours and negative (intrusive/aggressive and withdrawing and neglectful) behaviours simultaneously. Estimates of indirect effects were obtained using bootstrapping, the gold standard for testing indirect effects (Preacher \& Hayes, 2008). An indirect effect is significant if the $95 \%$ confidence interval (95\% CI) does not include zero. Model fit can be assessed using various indicators (Marsh, Hau, \& Wen, 2004; Raykov \& Marcoulides, 2006). Generally, the chi-square should be small and non-significant. The Comparative Fit Index (CFI) and TuckerLewis Index (TLI) should be above .90, whereas the Root Mean Square Error of Approximation (RMSEA) should be below .05.

\section{Results}

\section{Preliminary Analysis}

First we examined the distributions of infant attachment classifications. The four way distribution is reported in Table 1 and indicate that of the 88 infants in the sample 48 (55\%) were secure, $14(16 \%)$ were avoidant, $10(11 \%)$ were resistant, and $16(18 \%)$ were disorganized. The three way distributions (secure, insecure, disorganized) show that $48(45 \%)$ infants were classified as secure, 35 (35\%) infants were classified insecure and $16(18 \%)$ infants were 
classified as showing disorganized attachment strategies. In Table 1 we also report means of mothers' RF, mean sensitivity scores, as well as their mean scores on the disconnected and insensitive scales of the DIB for each type of infant attachment. Although we used the dimensional scores for disconnected and extremely insensitive behaviours in the path analyses, using the criteria of Out and colleagues (2009), 25\% of the mothers in our sample were classified as insensitive and $27 \%$ mothers were classified as disconnected.

Correlational analyses (see Table 2) show significant associations between maternal RF and both sensitivity and negative parenting, but not disconnected behaviours. Disconnected behaviours were only associated with insensitive behaviours. Infant attachment disorganization was associated with child sex, maternal education, maternal RF, and maternal insensitive behaviours. Infant attachment security was associated with maternal education and negative parenting.

\section{**INSERT TABLE 1 and 2 ABOUT HERE**}

To examine whether disconnected and extremely insensitive behaviours were associated with attachment, regression analyses using probit link were conducted. The results indicate that insensitive behaviours predicted attachment organization and security $(\mathrm{b}=-.63, \mathrm{SE}=.27, p<$ .05 , and $\mathrm{b}=-.49, \mathrm{SE}=.21, p<.05$, respectively). No association was found between disconnected behaviours and infant attachment organization or security $(b=-.07, \mathrm{SE}=.05, p>$ .05 , and $\mathrm{b}=-.07, \mathrm{SE}=.06, p>.05$, respectively). For this reason, we only focus on insensitive behaviours in the subsequent analyses.

There were no significant differences between mothers with histories of abuse and others in terms of RF, sensitivity, negative parenting or disconnected behaviours. Probit regressions 
also indicated no association between maternal history of abuse and infant attachment organization or security.

\section{Path analysis}

We then fitted a path analysis in which reflective functioning was allowed to predict both maternal behaviours and child attachment outcomes, whereas maternal behaviours predicted child attachment outcomes. A visual depiction of the model is presented in Figure 1. In the model, covariates (maternal education, maternal history of abuse and infant gender) were allowed to predict all variables in the model. Because the model is saturated, there is no test of model fit. Parameter estimates are reported in Tables 3 and 4. Maternal sensitivity was predicted by maternal education, whereas maternal insensitivity was only predicted by reflective functioning. Reflective functioning was predicted by maternal education. Infant attachment security was predicted by maternal education, as well as maternal insensitivity, whereas attachment organization was predicted by infant gender (boys being more likely to be disorganized; $28 \%$ of boys vs $9 \%$ of girls) and maternal insensitivity. The odds ratios indicate that an increase of 1 on the measure of negative behaviours increases the odds of attachment insecurity by 2.22 and attachment disorganization by 2.56 .

\footnotetext{
**Insert Figure 1 about here**
}

Next, we focused our attention on the results pertaining to the indirect association between mothers' reflective functioning and child attachment outcomes via maternal behaviours. Although the results are the same as those in Tables 3 and 4, we present them in Figure 1 for the sake of clarity. Reflective functioning was associated with maternal insensitivity but not 
maternal sensitivity. In turn, maternal insensitivity predicted both attachment security and attachment organization, consistent with potential indirect effects. We conducted a formal test of these indirect effects by running the same model with 5000 bootstrap samples. Results showed that the confidence intervals of the indirect effects between reflective functioning and both attachment security and attachment organization via maternal insensitivity did not include zero, $b$ $=.06,95 \% \mathrm{CI}[.002, .18]$ and $\mathrm{b}=.07,95 \% \mathrm{CI}[.01, .21]$ respectively. This indicates that the indirect effects are significant. In contrast, the confidence intervals of the indirect effects between reflective functioning and both attachment security and attachment organization via maternal sensitivity included zero, $\mathrm{b}=-.01,95 \% \mathrm{CI}[-1.60, .03]$ and $\mathrm{b}=.00,95 \% \mathrm{CI}[-.09, .06]$ respectively. This indicates that these indirect effects are not significant.

**Insert Table 3 and 4 about here**

\section{Discussion}

The study findings showed that mothers RF about attachment measured before the birth of the baby predicted their subsequent sensitivity and negative behaviours (intrusive aggressive and withdrawn). There was a pathway from mothers' RF to later infant attachment (both infant attachment security and infant attachment disorganization) through mothers' negative behaviours. These findings extend that of Fonagy, Steele, Steele, Moran and Higgitt (1991) that mothers' RF (on the AAI) was associated with infant attachment security and show that mother's RF is also associated with infant attachment disorganization. The findings that better mentalization is associated with maternal sensitivity is in line with the theory that there is an intergenerational pattern of transmission where mothers' RF, developed in the context of their own early attachment relationships, helps to make infant behaviour meaningful and underlies 
sensitivity. In addition, our findings suggest that better mentalization also helps mothers to screen their own negative intrusive, aggressive and withdrawn responses that undermine the development of attachment security and organization. Mothers with higher RF may be better at filtering their own affects of aggression, anxiety and fear, and screen their infants from negative behaviours, because they are more aware of their own affects and can see themselves from the outside and imagine the infants' distress. Mentalization also implies an implicit understanding that affects become less intense with time and can be changed through thinking and seeing situations differently, and this may help mothers with higher RF tolerating difficult feelings. At the same time, mothers who are not in touch with mental states may be more at risk of resorting to non-mentalizing, teleological actions where mental states are crudely translated into behaviours that ignore the infant's intentionality. Such behaviours may disrupt the infant's intentional actions, and disorientate the infant and undermine their developing self organization, whereas aggressive reactions and failure to respond may have a disorganizing impact though increasing anxious arousal.

We did not find an association between disconnected maternal behaviours and infant attachment disorganization. The lack of significant findings when comparing mothers of disorganized infants and others appears to be due to the fact that both mothers of insecure resistant/ambivalent infants had even higher rates of disconnected behaviours than mothers of disorganized infants. By comparison, mothers of secure infants and insecure avoidant infants displayed much lower rates of disconnected behaviours. Disconnected behaviours were also not significantly related to RF, which may in part be because the study lacked power. It may also be because these behaviours, for example, sexualized behaviours, operate in a way that is too far outside conscious awareness. Furthermore, disconnected behaviours have been shown to be 
specifically linked to lack of resolution of loss and trauma, involving dissociative processes (Hesse \& Main, 1999), and follows that it is more likely to be associated with RF regarding trauma and loss, rather than RF in general (Berthelot, et al., 2015).

Also, contrary to expectation, reported maternal histories of abuse were not associated with lower RF, or related to maternal sensitivity or negativity, or with an increased likelihood of their infant developing insecure or disorganized attachment. This is consistent with the findings of the Stacks et al., (2014) study where histories of abuse were also not associated with maternal RF about the child, parenting sensitivity or negativity, or infant attachment organization. In the present study, reflective functioning and maternal education, rather than abuse, were related to maternal sensitivity and maternal negative behaviours, as well as infant attachment security and organization. This extends previous findings that it is not trauma per se, but the capacity of the mother to mentalize about her early experiences that has implication for infant attachment security (Fonagy, Steele, Steele, Higgitt \& Target, 1994) or her capacity to resolve or mentalize trauma, that has implications for infant attachment disorganization (Berthelot, et al., 2015). While there is overwhelming evidence showing that childhood experiences of abuse is a risk factor associated with compromised parenting (Koren-Karie, et al., 2008), poor mental health, heightened stress reactivity, and poor affect regulation (Heim, Shugart, Craighead, \& Nemeroff, 2010), there is also evidence of resilience and individual and contextual protective factors (Dixon, \& Browne, 2009; Jaffee et al., 2013). Our findings and that of Stacks et al., (2014) suggest that in less stressful contexts they may be able to use their resources and relationships to limit the impact of trauma. This is also in line with the findings in the study by Fonagy et al., (1994) where mothers with histories of deprivation, but who had high reflective functioning, had securely attached infants. However, the way we assessed abuse in the current study as present or 
absent and using the AAI, rather than an validated measure of trauma, and the fact that we did not examine the severity of abuse and the type of trauma because of the relatively small subsample who reported abuse, may have contributed to the lack of significant findings regarding abuse. Furthermore, it is possible that mothers with histories of abuse experience more difficulties later, for example when confronted by the toddler's increasing strives for independence and self-assertion.

An unanticipated finding was that boys were at a significantly elevated risk of developing disorganized attachment styles; $28 \%$ of boys and $9 \%$ of girls in the sample displayed behaviours considered indicative of attachment disorganization during the SS. This adds to some previous observations that boys may be at higher risk for manifesting disorganized attachment patterns (Beebe \& Steele, 2013; Lyons-Ruth, et al., 1999; van IJzendoorn, 1995). However, David and Lyons-Ruth (2005) have suggested that boys are more likely to be classified as disorganized because they react with fight and flight responses when mothers behave in frightened or frightening ways, while girls show more "tend and befriend" reactions. Further longitudinal research is needed to ascertain whether such gender based differences in response styles are associated with risk over time or contribute to miss-classification.

With regard to mothers' RF and infant attachment, the findings of this study are broadly in line with that of Grienenberger and colleagues (2005) and Berthelot et al. (2015) that RF was associated with infant attachment organization. However, Grienenberger found a significant association only when infant attachment was considered ordinally as secure, insecure and disorganized, whereas we found clear associations with both attachment security and disorganization. Furthermore, while Grienenberger and colleagues (2005) assessed mothers' mentalization about the child using the Parent Development Interview (Slade, Grienenberger, 
Bernbach, Levy, \& Locker, 2005), we assessed mentalization regarding the mother's own early attachment relationships. It has been suggested that mentalization about the child is more proximal and directly relevant to the caregiving experience. However, our findings show that current mentalization about past attachment relationships and the parenting received, is not necessarily more distal, and may be particularly relevant when becoming a parent for the mother, and has important implications for mother-infant interaction, infant attachment security and organization. Like Grienenberger and colleagues (2005), we also found evidence for mediation, but in the present study negative intrusive and withdrawn behaviours mediated the relationship between mothers' RF and infant attachment disorganization, whereas in the Grienenberger study mediation was via affective communication errors. We did not assess affective communication errors, and the different mediating mechanisms may also reflect sample differences. Our sample was more heterogeneous in terms of income, education and reported abuse than the small and highly educated group of mothers in the Grienenberger study, and in our population we may have been able to observe a wider range of aggressive and intrusive behaviours. Contrary to our findings, Berthelot et al. (2015) did not report an association between RF about early attachment experience and infant secure or disorganized attachment in a sample of abused and neglected mothers. However, they found that reflective functioning regarding trauma assessed before the birth of the child predicted infant disorganized attachment. These different findings may also be related to the sample composition; while the sample in our study was composed of mothers from the community at low to moderate risk, the mothers in the study of Berthelot et al. (2015) all had histories of trauma.

As predicted, mothers' RF was also associated with sensitivity and this is in line with the findings of Stacks and colleagues (2014), although we focused on RF about early attachment 
relationships while they focused on RF about the child. We also predicted that mothers' RF, by helping to make infant behaviour meaningful, would be linked to sensitivity, which would in turn facilitate secure attachment. However, there was no evidence of a path through sensitivity and later infant attachment security in our sample, but this may be due to the fact that we assessed sensitivity at 6 months rather than concurrently. Our findings are thus divergent from that of Stacks (2014) where sensitivity, rather than parental negativity, mediated the relationship between maternal RF about attachment security, and where they found no association between $\mathrm{RF}$ and disorganization. Differences in the measures used to assess parenting sensitivity and negativity may have contributed to these divergent results given that the measure used in the present study to assess negative parenting behaviours, the DIP, was developed especially to assess behaviours that have previously been demonstrated to be linked with disorganization.

Both mother's RF and sensitivity were associated with education, in line with previous findings that maternal RF about the child was associated with education (Pajulo, et al., 2012) and that education was positively associated with maternal sensitivity (Tamis-Lemonda, Briggs, McClowry, \& Snow, 2009; Wendland et al., 2014), and negatively associated with extremely insensitive behaviours (Out et al., 2009). Education may be especially important when children do not have access in their own families to relationships that foster the development of mentalization and may be associated with the development of RF indirectly through access to relationships with teachers and peers outside the family that stimulate the development of RF.

While the study has several strengths such as the inclusion of a group of mothers who experienced childhood abuse, and the use of objective ratings of maternal behaviours in interaction with the infant, as well maternal RF, and infant attachment, the findings should be interpreted in light of certain limitations. First, the sample size is relatively small, and replication 
with a larger sample is required before drawing definite conclusions regarding the associations between RF, maternal behaviours, and infant attachment. Secondly, a weakness of the study is the fact that negative parenting was measured at the same time as infant attachment, whereas maternal sensitivity was measured when the infants were 6 months old, and may have contributed to finding that maternal insensitivity was relatively more important for infant attachment. The lack of significant associations between maternal histories of abuse and disconnected and insensitive behaviours was an unexpected finding, but replication with different and larger samples is necessary to investigate the implications of different types of abuse and abuse severity. Nearly all the mothers in the study were in common law relationships or married, and this is likely to have made an important contribution that was not considered in the present study. Further research is also needed to examine the infant's contribution to the interaction. Although more attention has traditionally been paid to the mother's role, there is evidence suggesting that infants also have an important role in maintaining or improving the quality of the interaction, for example by eliciting more positive responses from the mother (Kivijärvi et al., 2001). This may be due to genetic factors (Raby et al., 2012) and neurobiological differences (Tharner et al., 2011) that have been shown to increase the risk of attachment disorganization and which have not been assessed in the present study.

\section{Conclusion}

This study provides new evidence of a path from mothers' RF about their own early attachment relationships to infant attachment through parenting. This offers support for a model of intergenerational transmission where mothers' quality of mentalization in relation to early attachment relationships have implications for their ability to filter negative behaviours and develop and promote relationships that foster attachment security and organization. These 
findings further underscore the importance of a mentalizing stance for parenting. We propose that in the process of becoming a mother, it is not just the parent she wishes to be that is important, but also the quality of mentalizing about her own early attachment relationships. The mother's ability to access her experiences in mental state terms, to think about how it has been or should have been, also promotes her capacity to monitor her behavior and its impact on the infant. Difficulties in adopting a mentalizing stance regarding past and present relationships, might explain why mothers who manifest negative behaviours appear unaware of this even when it is evident to observers. In sum, the study provides evidence that mothers' mentalization about attachment relationships has important implications for their capacity to establish relationships where infants feel safe to explore and seek comfort. 


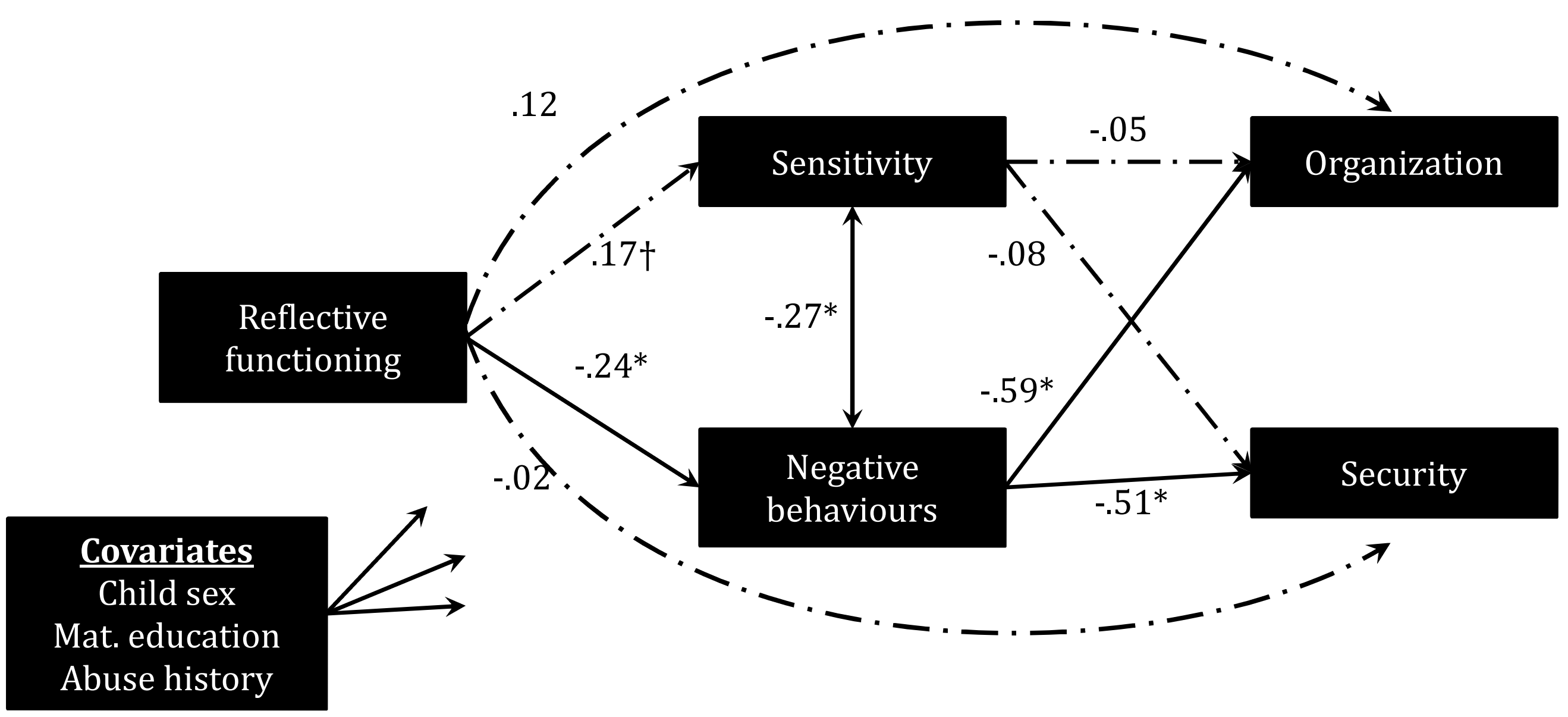

Figure 1. Path analysis describing the associations between reflective functioning, maternal behaviour and child attachment outcomes (attachment security and attachment disorganization). Covariates were allowed to predict all variables in the model (parameter estimates are presented in Tables 3 and 4). Parameters are standardized, except those predicting attachment security and attachment organization which are unstandardized estimates obtained using probit link. 
Table 1: Maternal reflective functioning and maternal behaviours according to child's attachment categories

\begin{tabular}{|c|c|c|c|c|c|c|c|c|}
\hline & \multicolumn{2}{|c|}{$\begin{array}{c}\text { Secure } \\
n=48(55 \%)\end{array}$} & \multicolumn{2}{|c|}{$\begin{array}{c}\text { Avoidant } \\
n=14(16 \%)\end{array}$} & \multicolumn{2}{|c|}{$\begin{array}{c}\text { Resistant/Ambivalent } \\
n=10(11 \%)\end{array}$} & \multicolumn{2}{|c|}{$\begin{array}{l}\text { Disorganized } \\
n=16(18 \%)\end{array}$} \\
\hline & $M$ & $S D$ & $M$ & $S D$ & $M$ & $S D$ & $M$ & $S D$ \\
\hline Maternal RF & 4.62 & 1.16 & 4.71 & 0.99 & 4.60 & 1.51 & 3.94 & 1.34 \\
\hline Disconnected behaviours & 1.98 & 2.46 & 1.79 & 2.58 & 3.50 & 3.14 & 3.06 & 2.98 \\
\hline Negative behaviours & 2.11 & 2.27 & 2.29 & 2.34 & 3.80 & 2.53 & 4.31 & 2.39 \\
\hline Sensitivity & 5.59 & 1.72 & 5.38 & 2.60 & 4.50 & 2.93 & 4.88 & 2.68 \\
\hline
\end{tabular}


Table 2: Correlational statistics for the main study variables ${ }^{1}$

\begin{tabular}{|c|c|c|c|c|c|c|c|c|c|c|}
\hline & 1 & 2 & 3 & 4 & 5 & 6 & 7 & 8 & $M$ & $S D$ \\
\hline \multicolumn{11}{|l|}{$\begin{array}{l}\text { 1. Child gender } \\
(1=\text { boys, } 2=\text { girls })\end{array}$} \\
\hline $\begin{array}{l}\text { 2. Abuse group } \\
(0=\text { no abuse }, 1=\text { abuse })\end{array}$ & .05 & & & & & & & & & 0.46 \\
\hline 3. Maternal Education & .12 & $-.25 *$ & & & & & & & 4.51 & 1.17 \\
\hline 4. Reflective Functioning & .05 & -.14 & $.25^{*}$ & & & & & & 4.52 & 1.26 \\
\hline 5. Negative Parenting & .09 & -.03 & -.18 & $-.24 *$ & & & & & 1.60 & 1.66 \\
\hline 6. Disconnected Behaviours & -.01 & -.20 & -.06 & -.03 & $.22^{*}$ & & & & 2.32 & 2.68 \\
\hline 7. Sensitivity & .04 & $-.18 \dagger$ & $.40^{*}$ & $.26^{*}$ & $-.32 *$ & -.08 & & & 5.92 & 1.42 \\
\hline $\begin{array}{l}\text { 8. Attachment Organization } \\
(0=\text { disorganized, } 1=\text { organized })\end{array}$ & $.25^{*}$ & -.11 & $.30 *$ & $.22 *$ & $-.27 *$ & -.11 & .17 & & & 0.39 \\
\hline $\begin{array}{l}\text { 9. Attachment Security } \\
(0=\text { insecure, } 1=\text { secure })\end{array}$ & $.20 \dagger$ & -.09 & $.32 *$ & .10 & $-.25^{*}$ & -.10 & .14 & $.52 *$ & & 0.50 \\
\hline
\end{tabular}

Note. $* p<.05, \dagger p<.10$

\footnotetext{
${ }^{1}$ Scores have been $\log$ transformed
} 
Table 3. Parameter estimates of the associations between covariates included in the path analysis and child attachment security and child attachment disorganization.

\begin{tabular}{|c|c|c|c|}
\hline & Estimate & S.E. & P-value \\
\hline \multicolumn{4}{|l|}{ Attachment organization regressed on } \\
\hline Child sex & $.84 *$ & .38 & .03 \\
\hline Maternal education & .25 & .17 & .14 \\
\hline Abuse history & -.13 & .47 & .77 \\
\hline Reflective functioning & .12 & .14 & .40 \\
\hline Negative parenting & $-.59 *$ & .25 & .02 \\
\hline Sensitivity & -.05 & .36 & .89 \\
\hline Attachment security regressed on & Estimate & & \\
\hline Child sex & $.51 \dagger$ & .27 & .06 \\
\hline Maternal education & .33 & .14 & .02 \\
\hline Abuse history & -.19 & .32 & .55 \\
\hline Reflective functioning & -.02 & .12 & .84 \\
\hline Negative parenting & $-.51 *$ & .19 & .02 \\
\hline Sensitivity & -.13 & .35 & .71 \\
\hline
\end{tabular}

Note. $* p<.05, \dagger p<.10$. Parameter estimates are unstandardized regressions using probit link. The regressions were estimated in the path analysis shown in Figure 1 but are reported here to avoid visual clutter. 
Table 4. Parameter estimates of the associations between covariates included in the path analysis and reflective functioning and parenting

\begin{tabular}{|c|c|c|c|}
\hline & Std Estimate & S.E. & P-value \\
\hline \multicolumn{4}{|l|}{ Sensitivity regressed on } \\
\hline Child sex & -.02 & .19 & .93 \\
\hline Maternal education & $.34^{*}$ & .10 & .00 \\
\hline Abuse History & -.07 & .10 & .49 \\
\hline Reflective functioning & $.17^{*}$ & .09 & .05 \\
\hline \multicolumn{4}{|c|}{ Negative parenting regressed on } \\
\hline Child sex & .26 & .21 & .22 \\
\hline Maternal education & -.16 & .11 & .18 \\
\hline Abuse History & -.11 & .12 & .29 \\
\hline Reflective functioning & $-.24^{*}$ & .10 & .02 \\
\hline \multicolumn{4}{|c|}{ Reflective functioning regressed on } \\
\hline Child sex & .13 & .21 & .56 \\
\hline Maternal education & $.23 *$ & .09 & .01 \\
\hline Abuse History & -.07 & .11 & .50 \\
\hline
\end{tabular}

\begin{tabular}{lc} 
Abuse History & -.07 \\
\hline Note. $* p<.05, \dagger p<.10$. We report standardized estimates obtained using linear regressions. The
\end{tabular}

regressions were estimated in the path analysis shown in Figure 1 but are reported here to avoid visual clutter. 


\section{References}

Afifi, T. O., Mather, A., Boman, J., Fleisher, W., Enns, M. W., Macmillan, H., \& Sareen, J. (2011). Childhood adversity and personality disorders: results from a nationally representative population-based study. Journal of Psychiatry Research, 45(6), 814822.

Ainsworth, M. D. S., Bell, S. M., \& Stayton, D. J. (1974). Infant-mother attachment and social development: "socialization" as a product of reciprocal responsiveness to signal. In M. P. M. Richards (Ed.), The integration of a child into a social world (pp. 99-135). London, U.K.: Cambridge University Press.

Ainsworth, M. D. S., Blehar, M., Waters, E., \& Wall, S. (1978). Patterns of attachment, a psychological study of the strange situation. Hillsdale: Lawrence Erlbaum.

Beebe, B., Jaffe, J., Markese, S., Buck, K., Chen, H., Cohen, P., ... Feldstein, S. (2010). The origins of 12-month attachment: a microanalysis of 4-month mother-infant interaction. Attachment \& Human Development, 12(1-2), 3-141.

Beebe, B., \& Steele, M. (2013). How does microanalysis of mother-infant communication inform maternal sensitivity and infant attachment? Attachment \& Human Development, 15(5-6), 583-602.

Bernier, A., \& Meins, E. (2008). A threshold approach to understanding the origins of attachment disorganization. Developental Psychology, 44(4), 969-982. doi: 10.1037/0012-1649.44.4.969

Berthelot, N., Ensink, E., Bernazzani, O., Normandin, L., Luyten, P., \& Fonagy, P. (2015). Intergenerational transmission of attachment in abused and neglected mothers: The role of trauma-specific reflective functioning. Infant Mental Health Journal, 36(2), 200-212. doi: 10.1002/imhj.21499

David, D. H., \& Lyons-Ruth, K. (2005). Differential Attachment Responses of Male and Female Infants to Frightening Maternal Behavior: Tend or Befriend Versus Fight or Flight? Infant Mental Health Journal, 21(1), 1-18.

Dixon, L., C.E., H.-G., \& Browne, K. D. (2009). Patterns of risk and protective factors in the intergenerational cycle of maltreatment. Journal of Family Violence, 24, 111-122.

Ensink, K., Berthelot, N., Bernazzani, O., Normandin, L., \& Fonagy, P. (2014). Another step closer to measuring the ghosts in the nursery: Preliminary validation of the Trauma Reflective Functioning Scale. Frontiers in Psychology, 5(1471), 1-12.

Fonagy, P., Steele, M., Steele, H., Moran, G. S., \& Higgitt, A. C. (1991). The capacity for understanding mental states: The reflective self in parent and child and its significance for security of attachment. Infant Mental Health Journal, 12(3), 201-218.

Fonagy, P., \& Target, M. (1997). Attachment and reflective function: their role in selforganization. Development and Psychopathology, 9(4), 679-700.

Fonagy, P., Target, M., Steele, H., \& Steele, M. (1998). Reflective-functioning manual, version 5.0, for application to adult attachment interviews. London: University College London.

George, C., Kaplan, M., \& Main, M. (1985). Adult attachment interview. University of California, Berkeley.

Grienenberger, J. F., Kelly, K., \& Slade, A. (2005). Maternal reflective functioning, motherinfant affective communication, and infant attachment: exploring the link between 
mental states and observed caregiving behavior in the intergenerational transmission of attachment. Attachment \& Human Development, 7(3), 299-311.

Heim, C., Shugart, M., Craighead, W. E., \& Nemeroff, C. B. (2010). Neurobiological and psychiatric consequences of child abuse and neglect. Developmental Psychobiology, 52(7), 671-690. doi: 10.1002/dev.20494

Hesse, E., \& Main, M. (1999). Second-generation effects of unresolved trauma as observed in nonmaltreating parents: Dissociated, frightened and threatening parental behavior. Psychoanalytic Inquiry, 19, 481-540.

Hesse, E., \& Main, M. (2000). Disorganized infant, child, and adult attachment: collapse in behavioral and attentional strategies. Journal of the American Psychoanalytic Association, 48(4), 1097-1127.

Isabella, R. A. (1993). Origins of attachment: maternal interactive behavior across the first year. Child Development, 64(2), 605-621.

Jaffee, S. R., Bowes, L., Ouellet-Morin, I., Fisher, H. L., Moffitt, T. E., Merrick, M. T., \& Arseneault, L. (2013). Safe, stable, nurturing relationships break the intergenerational cycle of abuse: a prospective nationally representative cohort of children in the United Kingdom. Journal of Adolescent Health, 53(4 Suppl), S4-10. doi: 10.1016/j.jadohealth.2013.04.007

Kivijärvi, M., Voeten, M. J. M., Niemelä, P., Räihä, H., Lertola, K., \& Piha, J. (2001). Maternal sensitivity behavior and infant behavior in early interaction. Infant Mental Health Journal, 22(6), 627-640.

Koren-Karie, N., Oppenheim, D., \& Getzler-Yosef, R. (2008). Shaping children's internal working models through mother-child dialogues: the importance of resolving past maternal trauma. Attachment \& Human Development, 10(4), 465-483. doi: $10.1080 / 14616730802461482$

Lyons-Ruth, K., Bronfman, E., \& Parsons, E. (1999). Atypical attachment in infancy and early childhood among children at developmental risk. IV. Maternal frightened, frightening, or atypical behavior and disorganized infant attachment patterns. Monographs of the Society for Research in Child Development, 64(3), 67-96.

Lyons-Ruth, K., \& Spielman, E. (2004). Disorganized Infant Attachment Strategies and Helpless-Fearful Profiles of Parenting: Integrating Attachment Research with Clinical Intervention. Infant Mental Health Journal, 25(4), 318-335. doi: 10.1002/imhj.20008

Madigan, S., Moran, G., \& Pederson, D. R. (2006). Unresolved states of mind, disorganized attachment relationships, and disrupted interactions of adolescent mothers and their infants. Developmental Psychology, 42(2), 293-304.

Main, M., \& Hesse, E. (1990). Parents' unresolved traumatic experiences are related to infant disorganized attachment status: Is frightened and/or frightening parental behavior the linking mechanism? In M. T. Greenberg, D. Cicchetti \& E. M. Cummings (Eds.), Attachment in the preschool years: Theory, research, and intervention (pp. 161-182). Chicago, IL: The University of Chicago Press.

Main, M., \& Hesse, E. (1998). Frightening, frightened, dissociated, deferential, sexualized and disorganized parental behavior: A coding system for parent - infant interactions (6th ed.). University of California at Berkeley.

Main, M., \& Solomon, J. (1990). Procedures for identifying disorganized/disoriented infants during the Ainsworth Strange Situation. In M. Greenberg, D. Cicchetti \& M. 
Cummings (Eds.), Attachment in the preschool years (pp. 121-160). Chicago: University of Chicago Press.

Marsh, H. W., Hau, K.-T., \& Wen, Z. (2004). In search of golden rules: Comment on hypothesis-testing approaches to setting cutoff values for fit indexes and dangers in overgeneralizing Hu and Bentler's (1999) findings. Structural Equation Modeling: A Multidisciplinary Journal, 11(3), 320-341.

Muthén, B. O. (2010). Bayesian analysis in Mplus: A brief introduction. www.statmodel.com/download/IntroBayesVersion\%203.pdf.

Muthén, L. K., \& Muthén, B. O. (1998-2012). Mplus User's Guide (Seventh edition ed.). Los Angeles, CA: Muthén \& Muthén

Out, D., Bakermans-Kranenburg, M. J., \& van IJzendoorn, M. H. (2009). The role of disconnected and extremely insensitive parenting in the development of disorganized attachment: validation of a new measure. Attachment \& Human Development, 11(5), 419-443.

Pajulo, M., Pyykkonen, N., Kalland, M., Sinkkonen, J., Helenius, H., Punamaki, R. L., \& Suchman, N. (2012). Substance-Abusing Mothers in Residential Treatment with Their Babies: Importance of Pre- and Postnatal Maternal Reflective Functioning. Infant Mental Health Journal, 33(1), 70-81. doi: 10.1002/imhj.20342

Preacher, K. J., \& Hayes, A. F. (2008). Asymptotic and resampling strategies for assessing and comparing indirect effects in multiple mediator models. Behavior Research Methods, 40(3), 879-891.

Raby, K. L., Cicchetti, D., Carlson, E. A., Cutuli, J. J., Englund, M. M., \& Egeland, B. (2012). Genetic and caregiving-based contributions to infant attachment: unique associations with distress reactivity and attachment security. Psychological Science, 23(9), 1016-1023. doi: 10.1177/0956797612438265

Raykov, T., \& Marcoulides, G. A. (2006). A First Course in Structural Equation Modeling: Taylor \& Francis.

Schechter, D. S., Coates, S. W., Kaminer, T., Coots, T., Zeanah, C. H., Jr., Davies, M., ... Myers, M. M. (2008). Distorted maternal mental representations and atypical behavior in a clinical sample of violence-exposed mothers and their toddlers. Journal of Trauma \& Dissociation, 9(2), 123-147.

Scher, C. D., Forde, D. R., McQuaid, J. R., \& Stein, M. B. (2004). Prevalence and demographic correlates of childhood maltreatment in an adult community sample. Child Abuse \& Neglect, 28(2), 167-180.

Slade, A., Grienenberger, J., Bernbach, E., Levy, D., \& Locker, A. (2005). Maternal reflective functioning, attachment, and the transmission gap: a preliminary study. Attachment \& Human Development, 7(3), 283-298.

Sroufe, L. A., Egeland, B., Carlson, E., \& Collins, W. A. (2005). The development of the person: The Minnesota study of risk and adaptation from birth to adulthood. New York: Guilford Press.

Stacks, A. M., Muzik, M., Wong, K., Beeghly, M., Huth-Bocks, A., Irwin, J. L., \& Rosenblum, K. L. (2014). Maternal reflective functioning among mothers with childhood maltreatment histories: links to sensitive parenting and infant attachment security. Attachment \& Human Development, 16(5), 515-533.

Suchman, N. E., DeCoste, C., Leigh, D., \& Borelli, J. (2010). Reflective functioning in mothers with drug use disorders: implications for dyadic interactions with infants and 
toddlers. Attachment \& Human Development, 12(6), 567-585. doi: 10.1080/14616734.2010.501988

Tamis-Lemonda, C. S., Briggs, R. D., McClowry, S. G., \& Snow, D. L. (2009). Maternal Control and Sensitivity, Child Gender, and Maternal Education in Relation to Children's Behavioral Outcomes in African American Families. Journal of Applied Developmental Psychology, 30(3), 321-331. doi: 10.1016/j.appdev.2008.12.018

Tharner, A., Herba, C. M., Luijk, M. P., van IJzendoorn, M. H., Bakermans-Kranenburg, M. J., Govaert, P. P., ... Tiemeier, H. (2011). Subcortical structures and the neurobiology of infant attachment disorganization: a longitudinal ultrasound imaging study. Social Neuroscience, 6(4), 336-347.

van IJzendoorn, M. H. (1995). Adult attachment representations, parental responsiveness, and infant attachment: a meta-analysis on the predictive validity of the Adult Attachment Interview. Psychological Bulletin, 117(3), 387-403.

van IJzendoorn, M. H., \& Bakermans-Kranenburg, M. J. (2006). DRD4 7-repeat polymorphism moderates the association between maternal unresolved loss or trauma and infant disorganization. Attachment \& Human Development, 8(4), 291307. doi: $10.1080 / 14616730601048159$

Wendland, B. E., Atkinson, L., Steiner, M., Fleming, A. S., Pencharz, P., Moss, E., ... Team, M. S. (2014). Low maternal sensitivity at 6 months of age predicts higher BMI in 48 month old girls but not boys. Appetite, 82, 97-102. 